\title{
Quality of life assessment in companion animals: what, why, who, when and how.
}

\section{Zoe Belshaw MA Vet MB PhD Cert SAM Dip ECVIM-CA AFHEA MRCVS}

\section{Abstract}

Quality of life is a commonly used phrase in veterinary medicine. It describes a complex evaluation that may be difficult for animals to perform, and the phrase "happiness" may be a more crude but useful approximation. Quality of life assessments should ideally be an integral part of our decision making, and should encompass evaluation of aspects of a pet's life beyond just its health. Assessments should aim both to evaluate an animal's quality of life, and to look for ways in which it might be improved. This article will discuss the challenges of assessing quality of life in companion animals, and will review the range of different methods available for assessment of quality of life in cats and dogs.

Keywords: Quality of life; assessment; end-of-life; euthanasia

\section{What do we mean by "quality of life"?}

The origins of the term "quality of life" extend back to Plato and Aristotle, who used it to explore the conditions needed for a "good" life (Zuna et al, 2009). The concept has since become widely adopted. Quality of life is measured in human patients to determine their success, and is used at a population level in discussions of the impact of behavioural and societal changes and policies. More recently, quality of life is increasingly applied to our veterinary species, for example when describing treatment benefits, as a research outcome measure and during euthanasia discussions.

There is broad consensus on a definition of quality of life in people. The World Health Organisation (WHO, 1996) defines quality of life as: "the individuals' perception of their position in life in the context of the culture and value systems in which they live and in relation to their goals, expectations, standards and concerns". In contrast, consensus has not been reached on how quality of life should be defined when applying the term to animals. Four key reasons for this are summarised below.

a) Quality of life is a highly individual construct

We know from our own friends and family that something which is extremely important to the life of one person may be of no consequence to another. Some people have huge goals and high expectations of themselves and those around them, others less so. Different people are also affected in different magnitudes by the same change in circumstances, for example the loss of a job or the birth of a child. Quality of life assessment in people therefore requires a complex, conscious, multi-factorial evaluation, and is best performed by the individual living that life. It is not known how well these factors may translate to animals, and even if an animal can conceptualise and appraise its own quality of life, as yet we have no way of measuring this directly.

b) A person's quality of life assessment changes with time

People adjust their attitude to, and appraisal of, their own circumstances over time - a concept known as "response shifting" (Sprangers and Schwartz 1999). For example, an individual who has suffered a catastrophic injury is likely to report a significant dip in their quality of life immediately afterwards. This decrease is typically maintained until their health condition reaches a position of relative stability. Subsequently, they may undergo a process described as recalibrating and 
reprioritising where they adjust what is important to them now. As a result, they may ultimately report their quality of life to be almost as good as it was before their injury, but the constituents that they describe as important may be radically different. In the same way, what is important for the quality of life of a teenager may be very different to that of the same individual when they are elderly. Evidence from human healthcare (Andresen et al 2001; Creemens et al 2006) suggests it is very difficult to reliable assesses another person's quality of life, or what impacts most affect their perception of it. As yet, we have very little insight into whether animals undergo a similar response shifting process after a change in their health, or as they age.

c) Quality of life encompasses more than health

The WHO (1996) definition demonstrates that quality of life is not just about an individual's health state. It is possible to be in good mental and physical health yet feel you have a poor quality of life, for example if you are lonely, feel unsafe in your own home, are facing significant financial challenges, or are in a job which is unsatisfying. For this reason, a separate term of "health-related quality of life" has been developed for use in human healthcare to specifically describe the impact of health on a person's overall quality of life. True quality of life is a composite measure that may take into account dozens, or even hundreds, of aspects of an individuals' past, present and anticipated future life. Again, application of this to animals remains challenging.

d) There is no clear cut-point for an "unacceptable" quality of life

The purpose of quality of life assessment, both in people and companion animals, is typically to provide information on which decisions can be based. In these assessments, quality of life is typically rated on a continuous scale from very low to very high. Due to the highly individual nature of quality of life and the response shifting phenomenon, defining a cut point at which quality of life can be deemed "unacceptable" is impossible at a population level, and is highly challenging even at an individual level (McMillan 2008).

Due to this complexity, defining quality of life for our veterinary species remains contentious. Definitions have been proposed, ranging from a suggestion by animal welfare scientist Donald Broom (2007) that quality of life is simply a "subset of welfare", to that by McMillan (2008) who suggested "quality of life is closely related to, and may be equivalent to, a number of other concepts such as well-being, welfare, happiness, life satisfaction and contentment". The challenge with both definitions is that they include reference to other terms which are also poorly defined.

It has been argued (Yeates 2013) that strict definitions are not needed. Indeed, a recent review by Belshaw et al (2015) identified that the term was rarely defined by authors of veterinary publications where canine quality of life was assessed. However, in the absence of a definition it is very difficult to know what has been assessed. For example, many of the assessments described in the review by Belshaw et al (2015) appeared to be health-related quality of life consequences of specific diseases, rather than quality of life in a broader sense. Definition of the term in relation to euthanasia decision making within a veterinary clinic may also be important, as owners may struggle to link the phrase with either measurable behaviours, or a clear idea of what is important to the individual animal in front of them (Belshaw 2017).

In the absence of a universally agreed definition for quality of life when applied to animals, it is important for both researchers and clinicians to think what they mean when they use the term themselves, and to explain that whenever the term is used. This author believes that the term "happiness", whilst itself difficult to specifically define and measure, may be a very useful approximation of what we are aiming to achieve, and assess, and may be a useful way of explaining 
the term to owners. It may also help to dissociate discussion of quality of life with euthanasia, which some owners may perceive as a threat.

\section{Why should we assess quality of life?}

Despite the challenges associated with defining quality of life, the broad concept is useful. Fundamentally, assessing quality of life should ensure that we see pets as a whole, rather than breaking them down into a series of functional or dysfunctional organ systems. It should encourage us to see each animal as an individual in how they are affected by illnesses and interventions, social interactions and changes in living conditions. This should then encourage us to consider the decisions we make at this individual, not disease cohort or population, level. It should also help us to look for ways that we can improve the quality of life of our patients in ways that extend beyond healthcare, both in the clinic and their home environment. Finally, it should stop us from extending life when that is not in the best interest of the individual patient in front of us.

\section{When should we assess quality of life?}

Quality of life discussions naturally occur when euthanasia is being considered as a means to determining when the individual's life is of sufficiently poor quality to justify ending it. This can be helpful in shifting the focus from specific health problems back to the whole animal. Considering the potential impact of a medical or surgical intervention on quality of life can also be a useful starting point when deciding whether that treatment option is right for each individual patient in front of us. However, quality of life assessment should ideally be a continual process throughout life, aimed at making the quality of life of every individual animal as good as possible through looking at what we provide for the animal, and how they are behaving.

\section{Who should assess quality of life?}

In humans, quality of life assessments are performed by the individual person where at all possible. Animal welfare scientists are working on methods that allow animals to tell us how they feel, but as yet these are not easy to use outside a research setting. For now, the best placed person to assess the quality of life of an animal is the person who knows that animal well. Assessments often rely on interpretation of the motivation and meanings of specific behaviours, so the people who spend most time with the animal may be best placed to make sense of why they do what they do. However, many owners are not experts in animal behaviour, and they may misinterpret or overlook particularly important behaviours. Combining structured owner report, clinical examination, and video clips of the behaviours in question that you can review together may give the best chance of an accurate insight.

\section{How can we assess quality of life?}

A wide range of assessment tools, typically in the form of questionnaires for owners to complete on a weekly basis, have been published by researchers aiming to assess quality of life in populations of animals. The purpose and quality of some of those developed for use in dogs are described in Yeates and Main (2009) and Belshaw et al (2015). A comprehensive review of all the tools available is beyond the scope of this article. The following section will instead summarise some of the advantages and disadvantages of taking different approaches, with some examples. The bottom line is that in all likelihood, something is better than nothing. It is likely that no single approach or tool will meet all the needs of any one clinician, so adoption of a range of approaches for different clients and scenarios may be most beneficial. Almost all assessments are reliant on owners' recall and interpretation of their animal's behaviour, and the relative reliability of this should be borne in mind. 
130 Recently, generic health-related quality of life assessments have been published for use in cats 131 (Freeman et al 2016, Tatlock et al 2017). However, by far the commonest published tools available 132 for quality of life assessment are those specific to an individual species and condition such as cancer 133 (Iliopoulou et al 2013, Lynch et al 2010, Vols et al 2017) and cardiac disease (Freeman et al 2005). 134 These tools typically ask disease focused questions such as "What is the impact of [condition $\mathrm{x}$ ] on 135 the animal's ability to run?". Some may also include a generic question, such as "Rate the animal's 136 quality of life on this 0-10 scale".

137 The advantage of these tools is that they provide a ready-made, structured set of questions which can be used within that specific population of animals. However, there are multiple disadvantages to using these health-related quality of life assessments in a clinic setting with individual patients. Due to the challenges of finding funding for this type of work, many of the published tools are only in the first rounds of validation so there may be little data available on how useful they actually are in either monitoring or aiding decisions. Notable exceptions include the tool developed by Noli and colleagues for canine (2011) and feline (2016) skin disease. Older animals may have multiple comorbidities, so using a single disease-specific scale may not be appropriate. Unpicking the effect of any disease on an animal's appetite, ability to run, sleep etc can be incredibly difficult given the whole range of other environmental factors that may also be influencing those behaviours. Many of the tools are for completion by the animals' owners, who may be biased in what they report if there is any fear that a negative assessment may lead to a euthanasia decision being made on their behalf. In addition, a "cut-off" both for treatment monitoring and making euthanasia decisions is not provided with these tools, so individual owner-clinician combinations need to decide how the scores will be used in decision making. Finally, the paper-based nature of these tools can make them impractical to use in a clinic setting where most records are now computerised. However, in certain circumstances they can be a useful way of monitoring changes over time and any assistance with decision making can be helpful.

\section{b) Generic/holistic quality of life assessment tools}

Quality of life assessments have also been developed that are not specific to health conditions. Often, these are simple questions such as "Rate your pet's quality of life in the past 7 days", rather than more complex tools. Whilst useful in raising the subject, it can be very hard to know both how to complete, and interpret, such an unfocused (and often undefined) question. Perhaps more useful are tools that provide a more holistic assessment of multiple factors that contribute to an animals' quality of life, as these may lead to identification of a range of different areas for improvement. The disadvantage of some paper-based versions of such tools (e.g. Mullan and Main 2007) is that they can run to multiple pages of questions about all aspects of an animals' life, making their completion by owners and interpretation by clinicians somewhat daunting in a clinic situation. However, they certainly have their place.

Recently, more innovative ways of collecting data have been developed, such as the online quality of life tool marketed by the NewMetrica company based in the UK, the use of which is described by Reid et al (2018). This tool encourages owners to collect data at home which an algorithm then converts into outputs relating to the dog's energy, happiness, comfort and calmness. A range of collar-mounted data collection devices are also now being marketed directly to owners by other companies, promising to collect and interpret data directly from the animals and send it to an app on the owners' telephone, and even into a veterinary practice management software system. Whilst 
these devices sound exciting, studies describing the reliability of the data collected, and the benefits to decision makers of having these data, have yet to be published.

\section{c) Quality of life discussion tools for use in a general practice setting}

Yeates et al (2011) published a prototype of an innovative tool that was designed to prompt review and discussion during a veterinary consultation of a how owners could improve a dog's quality of life. By asking owners to rate on a single line scale how well the dog's five welfare needs were being met, they were able to identify specific interventions for that dog. Subsequently, The People's Dispensary for Sick Animals have developed a similar tool called the Petwise MOT (PDSA 2018) that is now being used in bespoke consultations in all their clinics. Based on the five welfare needs, it adopts a traffic-lights system to alert owners to areas where they can improve the care for their pet. Training sessions are available from the PDSA to learn how to use this method in other practice settings. The advantages of both these initiatives is that they are designed for use in clinics, can be used on any animal at any stage in their lives, and they strive for improvement rather than monitoring decline. The main criticism might be their relatively limited scope which may restrict the topics discussed.

\section{d) No-tool assessments}

Most of the discussions relating to quality of life in a clinical setting use no tools at all, relying simply on a discussion between vet and owner about the animal in front of them. This ensures that there are no distractions from inaccurate or irrelevant data, and no challenges of interpreting or making decisions on the basis of numeric scales. However, it appears that these discussions may be prompted more by owners than vets, and may centre mainly around euthanasia decisions rather than proactive quality of life improvement initiatives at an earlier stage (Belshaw 2017). In addition, common phrases used during these discussions such as "You'll know when the time is right" may not be helpful, or true.

There is some evidence that more structured conversations and assessments may be helpful. Christiansen et al (2016) described interviews with Danish owners of chronically ill pets, some of whom would have liked more support from their vet in making difficult decisions, particularly around euthanasia. Asking owners to identify specific behaviours to monitor may be helpful. Looking at the non-physiologically driven choices that animals make (e.g. play, sleeping in the sun, sniffing, purposeful interactions with people and other animals) may give the best insight into how they are feeling and may help to shift the focus away from less helpful behaviours of survival (e.g. eating, drinking, walking, toileting). Encouraging owners to facilitate as much as possible the activities that their pet enjoys, and to monitor their response to different stimuli which they usually enjoy may provide them with a useful framework to assess both the success of interventions, and to make euthanasia decisions.

Proactively asking owners to collect photos or video clips on their telephone of their pet doing different activities around their house can also provide hugely useful insights into what is happening in the home environment and can overcome misinterpretations of common behaviours. Videos and photos may highlight simple areas where improvements can be made, and by serially videoing the pet performing the same activities or on the same walk, subtle deteriorations can be detected that may otherwise be overlooked by an owner who sees the animal all the time.

Ideally, quality of life discussions should not be restricted to animals reaching the end of their lives. Serially engaging with owners to review how happy their animal is, and how they might be able to make it happier through what they provide for it, how they interact with it, and what they permit it 
to do would be a useful part of every single consultation. Each national government within the UK has produced resources for owners explaining their duty of care as relates to the Animal Welfare Act (2006), but awareness of the Act is reportedly low in the pet owning population (PDSA 2017). The Scottish Code of Conduct for the Welfare of Dogs (Scottish Government 2011a) and cats (Scottish Government 2011b) are particularly comprehensive and provide some excellent, specific guidance on meeting the welfare needs of these species in all aspects of their lives. Simply ensuring all owners know that these documents exist and encouraging them to read them would be a huge step forward.

\section{Conclusions}

Quality of life is a complex concept to apply to animals. Nevertheless, the broad sentiment is important and should be a central part of all the decisions we make as clinicians. Quality of life assessment can be performed in a wide range of ways and different methods may suit different clients and animals. Assessments should aim both to monitor, and to seek to improve each animal's quality of life. Many of the tools available have been developed for specific health-related research purposes and few are optimised for use in a 10-15 minute consultation. The PetWise MOT and the new technology-based home monitoring systems are exceptions to this. Simply raising the topic of quality of life and discussing what it means to the animal in front of you can be incredibly helpful for owners. Simple interventions such as highlighting to owners the useful guidance in the national codes of welfare conduct or asking them to bring in photos or videos of their pet's home environment could lead to big improvements in the quality of life of individual patients. This is a very active research field, and new tools are launched every year so keep an eye on the literature for advances.

\section{Key points (3-5)}

- The concept of quality of life is difficult to apply to animals, but using terms such as "happiness" may be a useful approximation

- Quality of life assessment should not be restricted to euthanasia decision making, but instead monitored and optimised through each animal's life. Use assessments that aim to improve an animals' quality of life rather than monitoring it through decline until the point that euthanasia is deemed necessary.

- A wide range of formats of quality of life assessment exist. None are perfect, but all have their place. Doing something is likely to be better than doing nothing.

- Engaging owners in collecting video clips and photos in the home environment that can be reviewed in the clinic may provide incredibly useful insights into both how the animal is doing, and what might be improved.

\section{References}

Andresen EM, Vahle VJ, Lollar D (2001) Proxy reliability: Health-related quality of life (HRQoL) measures for people with disability. Qual Life Res 10(7): 609-619

Belshaw Z, Asher LA, Harvey ND, Dean RS. (2015) Quality of life assessment in dogs: An evidencebased rapid review. Vet Journal 206(2): 203-12

Belshaw Z. (2017) Decision making and welfare assessment in canine osteoarthritis. PhD Thesis. http://eprints.nottingham.ac.uk/42077/ (accessed 22 February 2018). 
Broom, D. (2007) Quality of life means welfare: How is it related to other concepts and assessed? Anim Welf 16(S): 45-53

Christiansen ZB, Kristensen AT, Lassen J, Sandoe P (2016) Veterinarians' role in clients' decisionmaking regarding seriously ill companion animal patients. Acta Vet Scand 58:30.

Creemens J, Eiser C, Blades M (2006) Factors influencing agreement between child self-report and parent proxy-reports on the Pediatric Quality of Life Inventory ${ }^{\mathrm{TM}} 4.0$ (PedsQL ${ }^{\mathrm{TM}}$ ) generic core scales Health Qual Life Outcome 4: 58

Freeman LM, Rush JE, Farabaugh AE, Must A. (2005) Development and evaluation of a questionnaire for assessing health-related quality of life in dogs with cardiac disease. J Am Vet Med Assoc 226(11): 1864-8.

Freeman LM, Rodenberg C, Narayanan A, Olding J, Gooding MA, Koochaki PE (2016) Development and initial validation of the Cat HEalth and Wellbeing (CHEW) Questionnaire: a generic healthrelated quality of life instrument for cats. J Fel Med Surg 18(9): 689-701

272 Iliopoulou MA, Kitchell BE, Yuzbasiyan-Gurkan V. (2013) Development of a survey instrument to 273 assess health-related quality of life in small animal cancer patients treated with chemotherapy. J Am 274 Vet Med Assoc 242 (12): 1979-87. Lynch S, Savary-Bataille K, Leeuw B, Argyle DJ. (2011) Development of a questionnaire assessing health-related quality-of-life in dogs and cats with cancer. Vet Comp Onc. 9 (3): 172-82.

McMillan FD (2008) The concept of quality of life in animals In: McMillan FD, ed. Mental Health and Well-being in Animals, Wiley Blackwell, Chichester UK. p.185

Mullan S, Main D (2007) Preliminary evaluation of a quality-of-life screening programme for pet dogs. J Small Anim Pract 48 (6): 314-22

Noli C, Minafo G, Galzerano M (2011) Quality of life of dogs with skin diseases and their owners: Part 1: Development and validation of a questionnaire. Vet Dermatol. 22, 344-51. evaluate the Quality of Life of cats with skin disease and their owners, and its use in 185 cats with skin disease. Vet Dermatol. 27 (4): 247-57 PDSA (2017) The People's Dispensary for Sick Animals (PDSA) Animal Wellbeing (PAW) Report; PDSA, Telford, UK.

PDSA (2018) What are Petwise MOTs? https://www.pdsa.org.uk/education-centre/petwisemot/what-are-petwise-mots (Accessed 23rd February 2018)

Reid J, Nolan A, Scott M (2018) When is the right time? Vet Record 182: 85-6.

Scottish Government (2011a) Code of Practice for the Welfare of Dogs

http://www.gov.scot/Resource/Doc/304660/0095599.pdf (Accessed 23rd February 2018)

Scottish Government (2011b) Code of Practice for the Welfare of Cats 
297 Tatlock S, Gober M, Williamson N, Arbuckle R. (2017) Development and preliminary psychometric

298 evaluation of an owner-completed measure of feline quality of life. Vet J. 228:22-32

299 Vøls KK, Heden MA, Kristensen AT, Sandøe P. (2017) Quality of life assessment in dogs and cats

300 receiving chemotherapy - a review of current methods. Vet Comp Onc. 15(3):684-91

301 World Health Organisation (1996) WHOQOL-BREF: Introduction, administration, scoring and generic

302 version of the assessment http://www.who.int/mental health/media/en/76.pdf (accessed 22

303 February 2018)

304 Yeates J, Main DM (2009) Assessment of companion animal quality of life in veterinary practice and 305 research. J Small Anim Pract 50: 274-81

306 Yeates JW, Mullan S, Stone M, Main DC. (2011) Promoting discussions and decisions about dogs'

307 quality-of-life. J Small Anim Pract 52 (9): 459-63

308 Yeates J. (2013) Animal welfare in veterinary practice. $1^{\text {st }}$ edition. Wiley Blackwell. Chichester, UK.

309 Zuna NL, Turnbull A, Summers JA (2009) Family quality of life: moving from measurement to

310 application. J Pol Pract Intel Disability 6: 25-31 